\title{
Epithermal Neutron Radiography and Tomography on Large and Strongly Scattering Samples
}

\author{
Burkhard Schillinger ${ }^{1, a^{*}}$, Aaron Craft ${ }^{2, b}$ \\ ${ }^{1}$ Heinz Maier-Leibnitz Zentrum and Physics E21, Technische Universität München, \\ Lichtenbergstr.1, 85748 Garching, Germany \\ ${ }^{2}$ Advanced Characterization and Post-Irradiation Examination Department Idaho National \\ Laboratory, USA \\ a Burkhard.Schillinger@frm2.tum.de , ${ }^{\mathrm{b}}$ Aaron Craft@inl.gov,
}

Keywords: Neutron Imaging, Neutron Computed Tomography, Cadmium Filter, Epithermal Neutrons, Penetration

\begin{abstract}
Standard neutron imaging is usually performed with a thermal or cold spectrum, but sometimes, penetration of thermal or cold neutrons is not sufficient for thick samples, so higher energies should be tried e.g. on technical machine parts or on rock samples containing crystal water. A 1-2 mm cadmium filter effectively absorbs all neutrons with energies lower than 0.4 $\mathrm{eV}$, but is relatively transparent for higher energies, including epithermal neutrons. Radiography and computed tomography measurements were recorded at Idaho Nation Laboratory in the USA and Heinz Maier-Leibnitz Institut (MLZ) at the FRM II reactor in Germany. Image quality was improved by better penetration and by filtering out scattered and thermalized neutrons.
\end{abstract}

\section{Introduction}

Standard neutron imaging is usually performed with a thermal or cold spectrum, but large samples may either attenuate these energies too much, or generate too much scattering that blurs the obtained images. This is illustrated exemplarily on an electric motor and pump worth thick steel parts, and with a ferrous rock sample containing crystal water. To alleviate this problem, higher energies should be tried. Instead of fission or fusion neutrons of several $\mathrm{MeV}$, the epithermal energy range above the so-called cadmium edge can be tried.

A 1-2 mm cadmium filter cuts off all neutrons with energies lower than $0.4 \mathrm{eV}$ (Fig.1a) [1], but is relatively transparent for higher energies. For energies just above $0.4 \mathrm{eV}$, a standard $6 \mathrm{LiF}+\mathrm{ZnS}$ neutron screen can be used, as opposed to ZnS screens that use an organic binder to generate recoil protons triggered by fast $(\sim 1 \mathrm{MeV})$ neutrons. But since the $6 \mathrm{Li}$ cross section is inversely proportional to the neutron velocity and as such follows the $1 / \mathrm{v}$ law (Fig.1b) [1], the visible neutron energies may be in the range $0.4-100 \mathrm{eV}$, rather lower than higher, as sensitivity decreases with increasing energy. Radiography and computed tomography measurements were recorded at Idaho Nation Laboratory (INL) in the USA and Heinz Maier-Leibnitz Institut (FRM II) in Germany. Image quality was improved by better penetration through the sample and by filtering out down-scattered and thermalized neutrons behind the sample. 

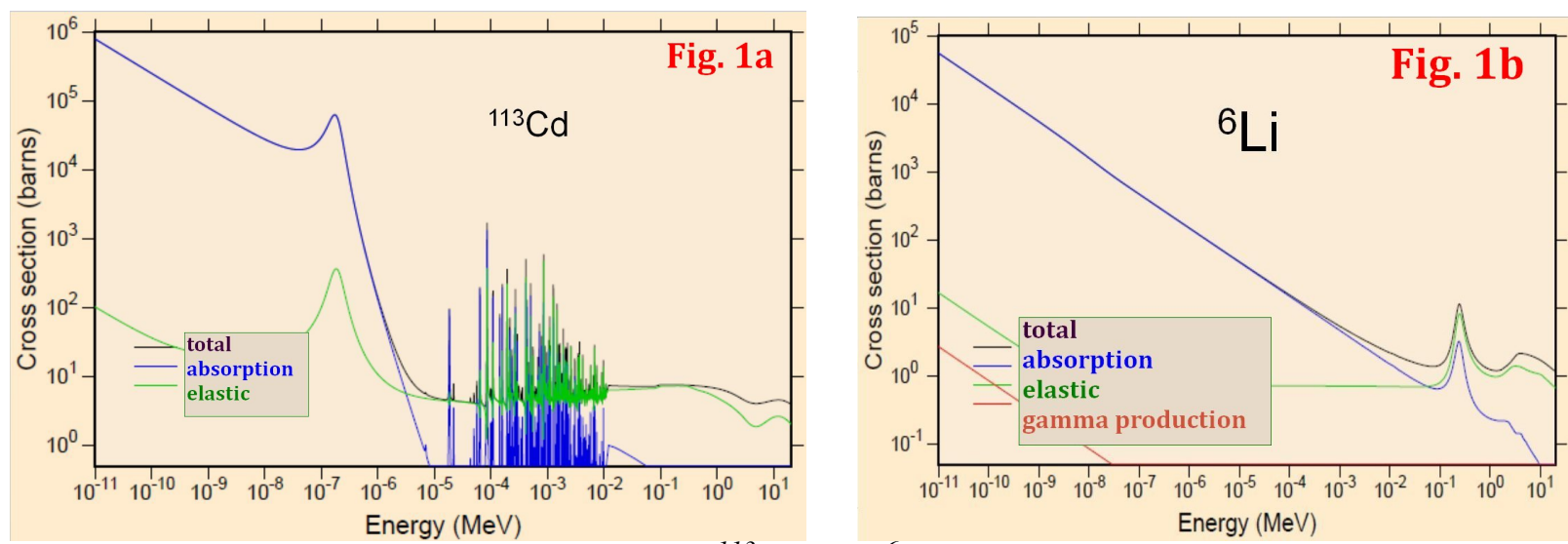

Fig. 1a,b: Neutron cross sections for ${ }^{113} \mathrm{Cd}$ and ${ }^{6} \mathrm{Li}$ from the Los Alamos database [1]

\section{Measurements at the NRAD reactor of Idaho National Lab}

The $250 \mathrm{~kW}$ NRAD reactor at INL is optimized for radiography on highly radioactive samples (spent fuel) using the transfer method with dysprosium and cadmium-filtered indium foils, which are sensitive in the thermal and epithermal ranges, respectively [2]. For this, the beam tubes are mounted close to the cladding of the core (Fig.2) with only a few millimeters of water between the fuel and beginning of the beam tube, so the epithermal flux is maximized because only minimal neutron moderation happens on the way into the beam tubes. The thermal flux, if unfiltered, is still high enough for standard thermal neutron imaging. The neutron energy spectrum is shown in Fig. 3 [3]. Because of the high radiation level in the measuring chamber, electronic detectors were introduced only very recently [4], [5]. For the measurements, a $1 \mathrm{~mm}$ Cd filter was placed before the sample (Fig.4), and for the double-filtered measurements described below, a second filter was placed between sample and detector.
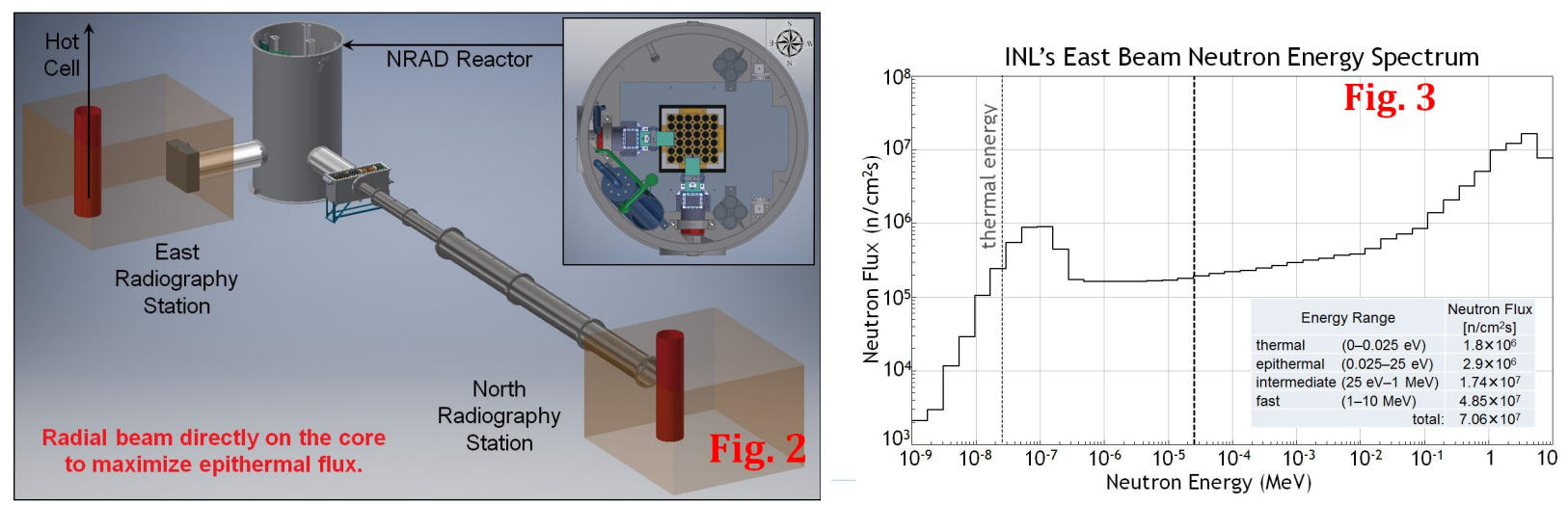

Fig. 2,3: The NRAD radiography facilities and the neutron spectrum at NRAD

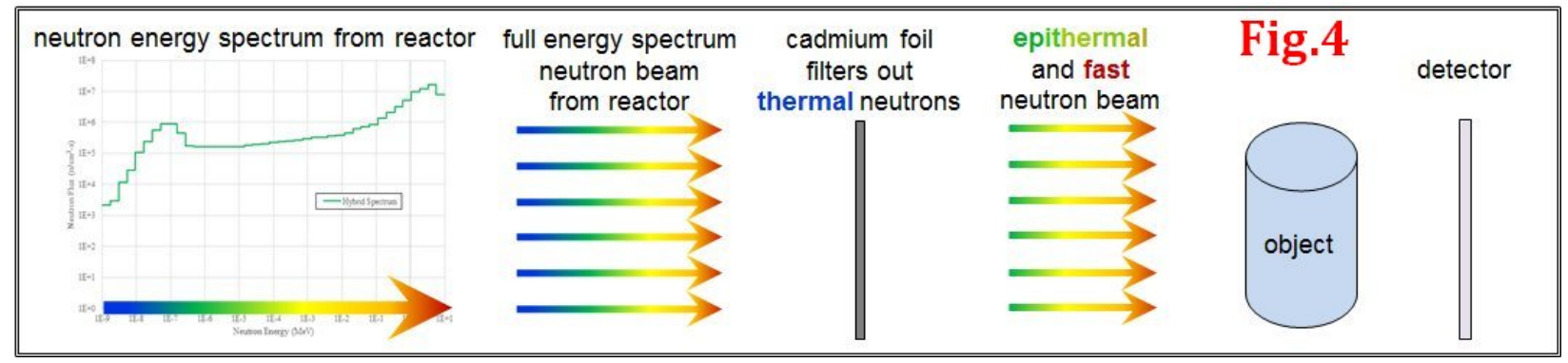

Fig. 4: Filtering the beam with Cd 
Fig. 5a and 5b show epithermal measurements at INL. Due to the low sensitivity of the ${ }^{6} \mathrm{LiF}+\mathrm{ZnS}$-screen for epithermal neutrons, exposure time was 10 minutes. A fist-sized rock containing iron and a lot of crystal water was impenetrable for thermal neutrons, and also for epithermal neutrons, it showed an unclear and smeared radiography image. This is of special interest because many fossils are found in this type of rock, which is impenetrable for X-rays. A brass screw placed behind the rock became visible only after a second Cd filter was placed between sample and detector - the crystal water had apparently downscattered and thermalized the epithermal neutrons, which could then be absorbed by the second Cd filter. Fig 5b proves that the image truly shows epithermal neutrons - the Siemens star in its holder, made from Gadolinium, is completely transparent. A thermal radiography is shown further down with the MLZ measurements. The boron nitride discs in the Beam Purity Indicator on top attenuate much more than the lead discs, proving it is mainly a neutron, not a gamma image. A $1 \mathrm{~cm}$ stripe of lithiated polyethylene attenuates more than $1 \mathrm{~cm}$ of lead.

The employed LiF+ZnS scintillation screen is not sensitive to fast fission neutrons, so they remain unused in this setup, but first tests with a fast neutron screen are promising.

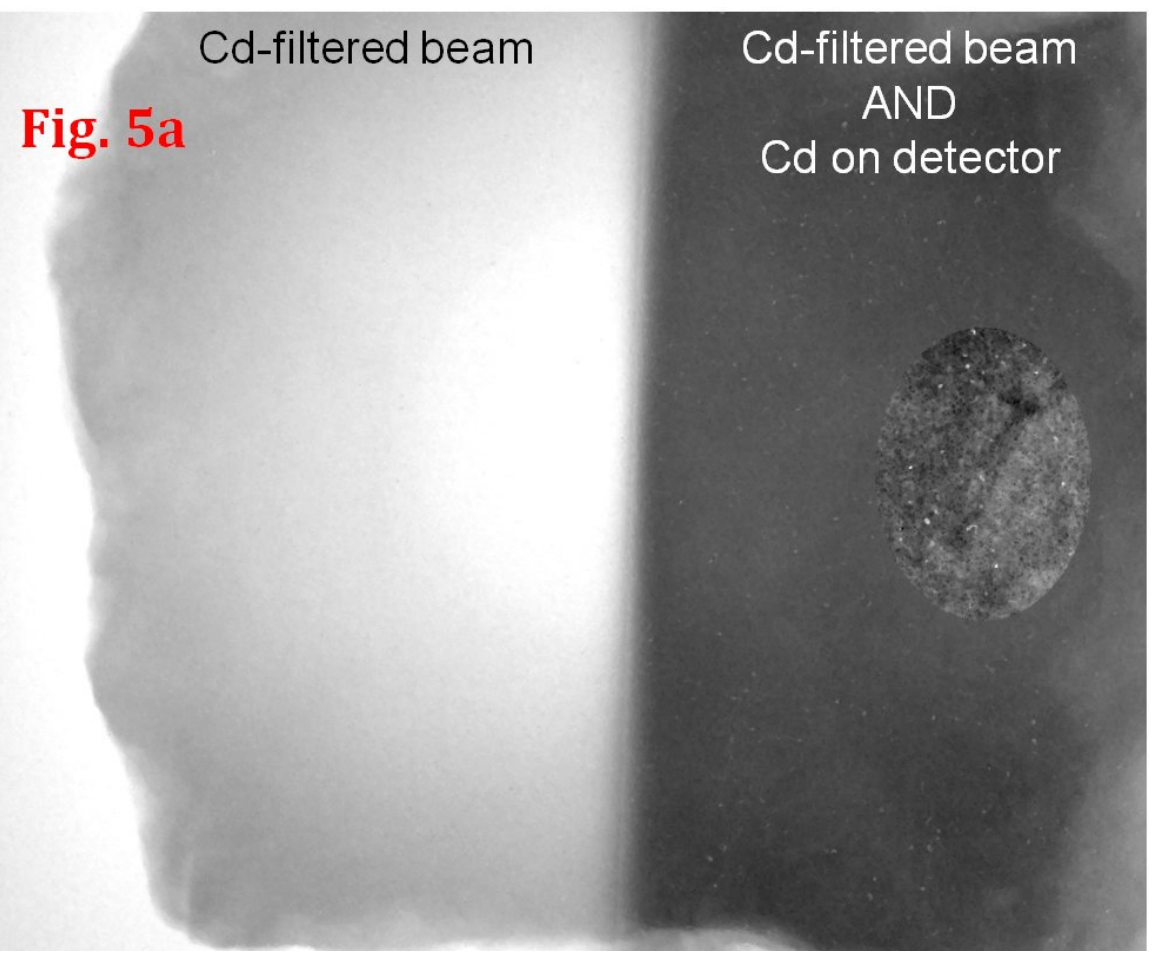

Fig. 5a: Fist-sized ferrous rock with crystal water single and double Cd-filtered. The image is enhanced in the oval showing a brass screw behind the rock. 


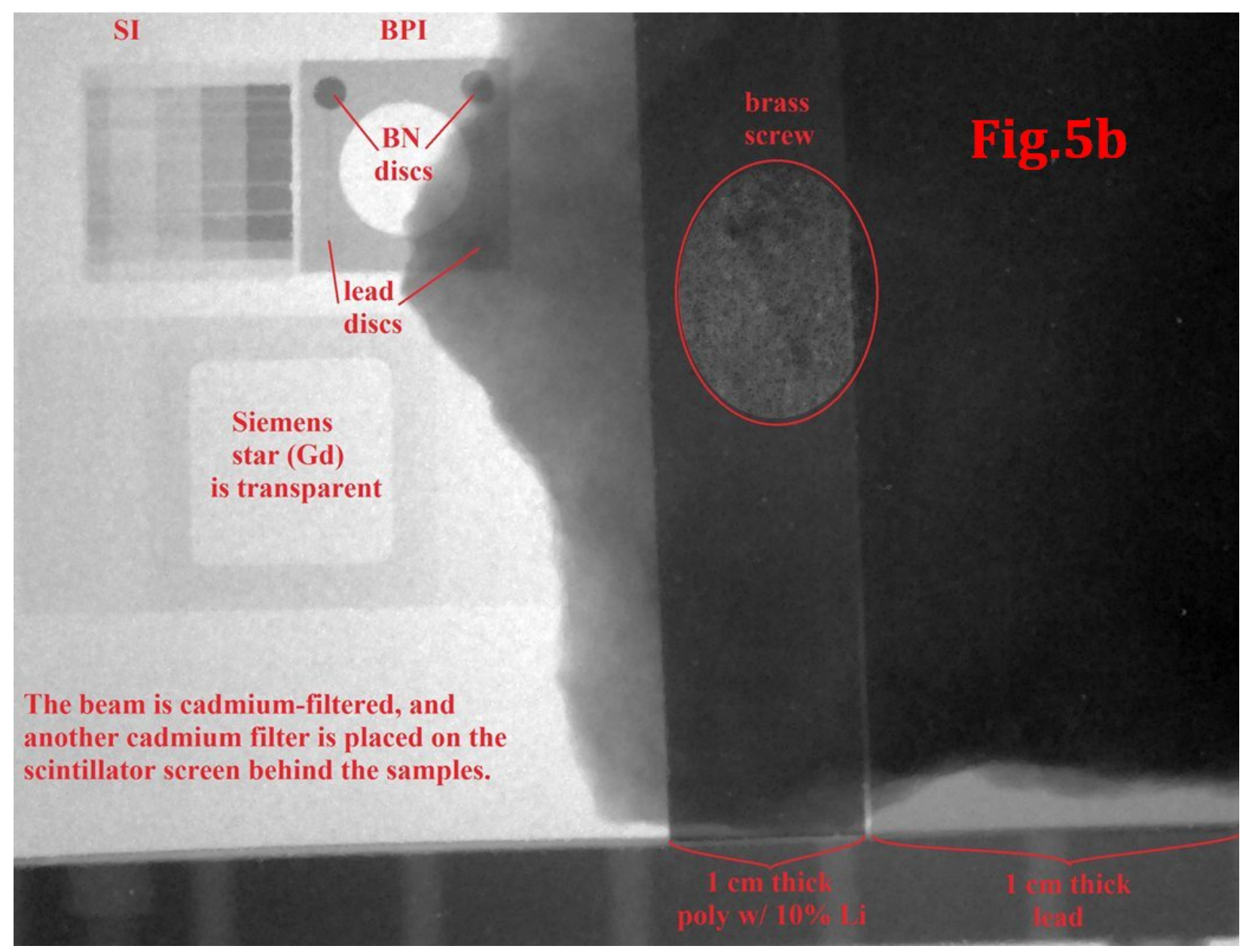

Fig. 5b: Fist-sized ferrous rock with crystal water single and double Cd-filtered with additional samples of Gd, lead and lithiated polyethylene.

\section{Measurements at the FRM II reactor of Heinz Maier-Leibnitz Zentrum at Technische Universität München}

The 20 MW FRM II reactor uses a compact core and provides the highest neutron flux per MW worldwide. For tumor treatment and fast neutron imaging, FRM II has a converter plate made of uranium fuel that can be moved in front of a beam tube to produce extra unmoderated fission neutrons (Fig.6). With $80 \mathrm{~kW}$ power of the converter plate, a third of the epithermal output of NRAD at INL was expected at the NECTAR neutron imaging facility, but unfortunately, the gamma flux at this facility is so high that in a Cd-filtered beam, polyethylene appears transparent, and lead appears dark, so the resulting images are gamma images, even in spite of the low gamma sensitivity of the $\mathrm{LiF}+\mathrm{ZnS}$ screen.

The only alternative is the cold neutron imaging facility ANTARES, which looks directly at the cold source, so no epithermal flux was expected. However, the cold source is optimized for maximum output of cold neutrons, so it is undermoderated, because a fully thermalized cold source would have too much self-absorption. Surprisingly, a significant amount of epithermal neutrons penetrates through the cold source, so epithermal radiographs need only one minute of exposure time. Fig. 7 shows a thermal and an epithermal radiography of a Gd Siemens star. Gd is transparent for epithermal neutrons, so it remains invisible. 


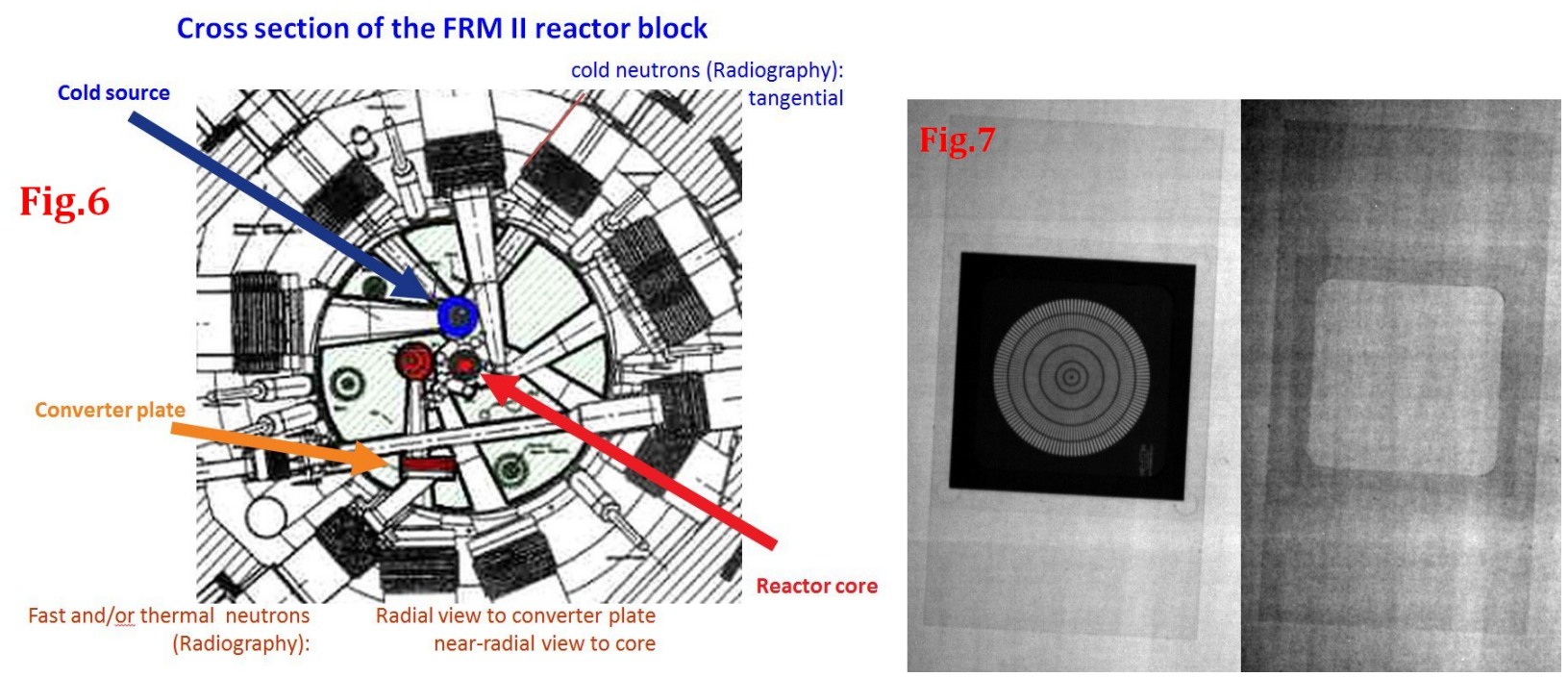

Fig. 6 (left): Cross section of the FRM II reactor block, Fig.7 (right): A thermal and an epithermal radiograph of a Gd Siemens star. Gd is transparent for epithermal neutrons.

The experiment with the ferrous rock was also repeated at FRM II. Fig.8 shows the singlefiltered image of a rock with a plastic sleeve and a brass screw and bolt on the left, while the double-filtered image on the right shows increased contrast for the small samples. If the distance between the detector and the samples is increased, the contrast for the small samples also increases in the single-filtered image, which proves that the blurring is caused by scattered neutrons which go in all directions, and have less chance of hitting the detector in increased distance.
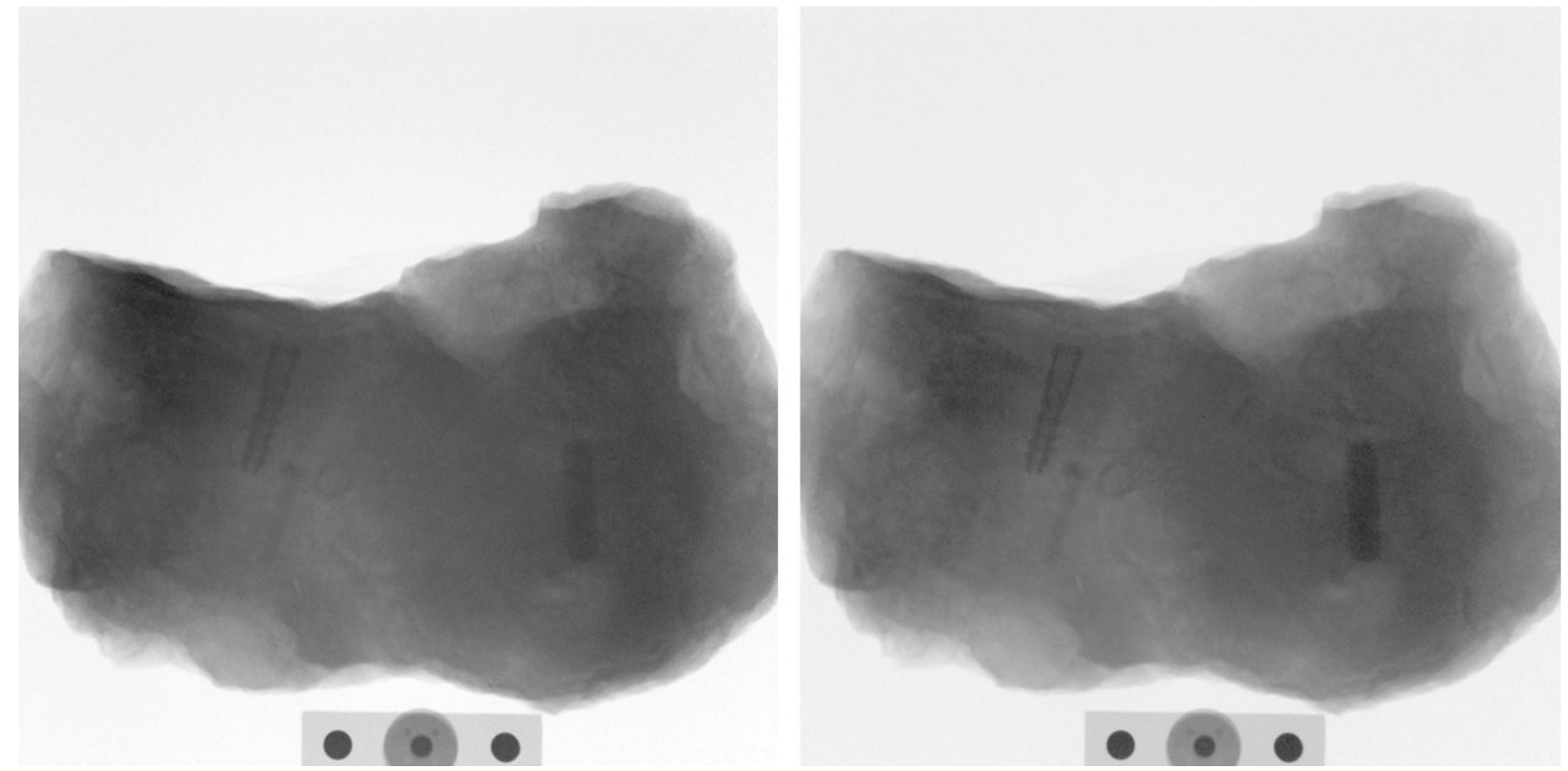

Fig. 8: The ferrous rock experiment repeated with one (left) and two (right) Cd filters: The double-filtered image shows increased contrast for a plastic sleeve end a brass screw and bolt.

With only 60 seconds for one epithermal image, the intensity was sufficient to perform epithermal computed tomography of a technical sample, in this case an electric motor with an 
attached water pump. The material thickness of the pump can in some places not even be penetrated by epithermal neutrons, but on the whole, penetration is much better than for cold neutrons, i.e. without the Cd filter. Fig. 9 shows a cold and an epithermal radiography of the pump, with a small photograph of the setup top center. The steel of the electro magnet of the motor and parts of the impeller of the pump remain impenetrable even for epithermal neutrons, but the rest shows better penetration for epithermal neutrons.

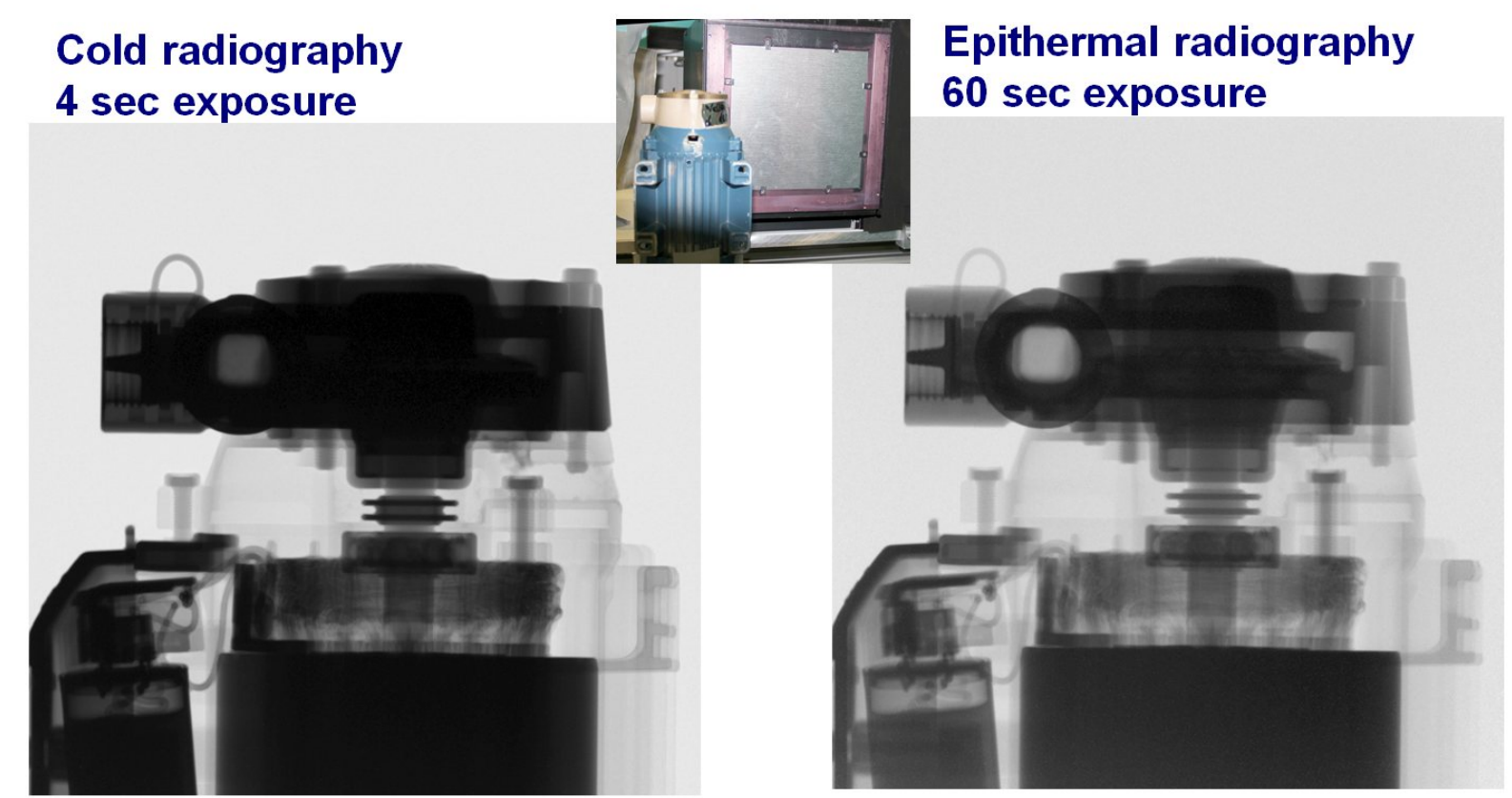

Fig. 9: Cold and epithermal neutron radiography of an electric motor and pump.

In the reconstruction in Fig. 10 and 11, two tomographic slices are shown each for cold and epithermal neutrons. For cold neutrons, the pump impeller is not reconstructed at all, for epithermal neutrons, it works at least in part. For regions of good penetrability, there is no loss in resolution visible for epithermal neutrons compared to cold neutrons, as can be seen in the 3D views in Fig. 12.
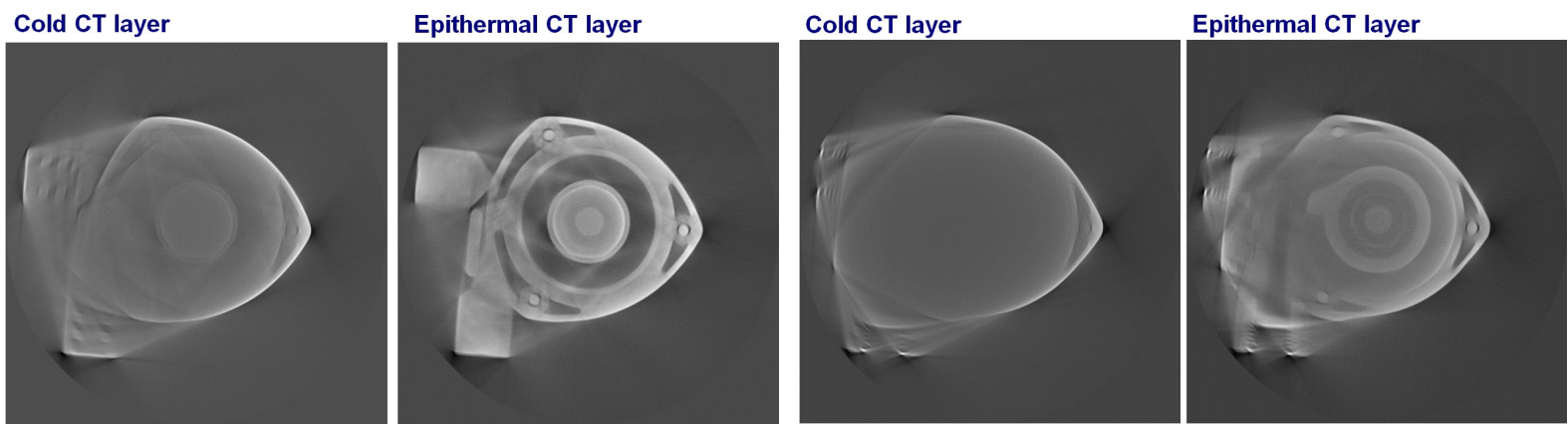

Fig. 10 (left two) and 11 (right two): Two tomographic slices each for cold and epithermal neutrons. For cold neutrons, the pump impeller is not reconstructed at all, but it works to some extent for epithermal neutrons. 


\section{Cold 3D CT}

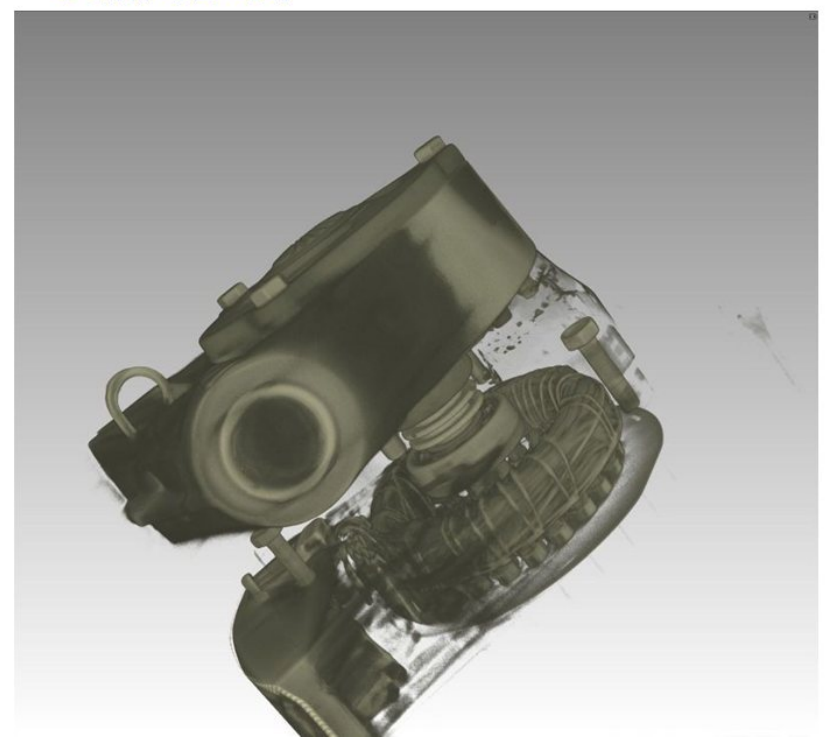

Epithermal 3D CT

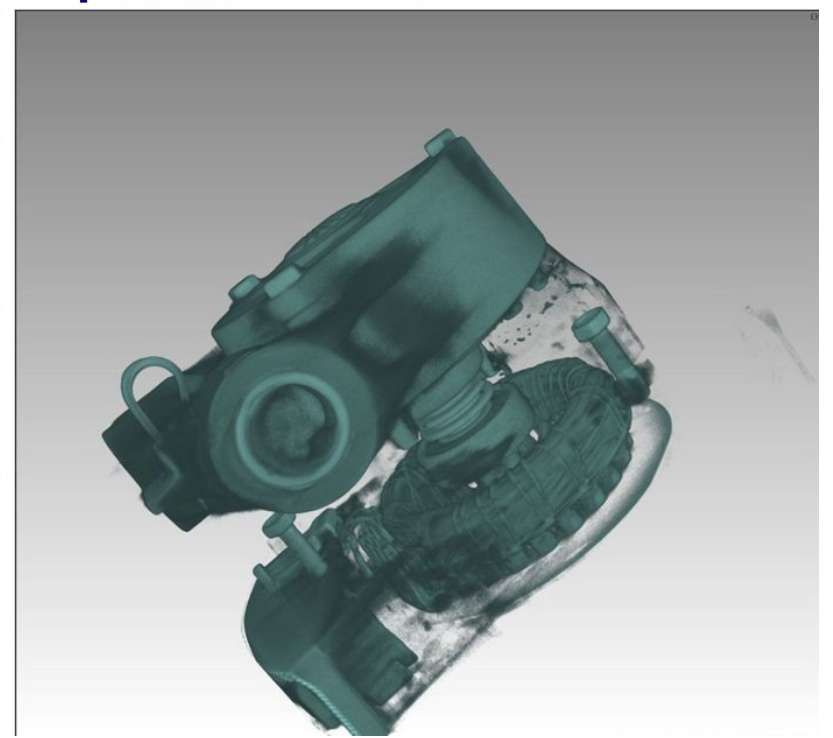

Fig. 12: 3D views of the cold end epithermal neutron CT reconstruction

\section{Conclusions and Outlook}

Epithermal neutron imaging will perform no wonders, but can significantly increase penetrability on thick samples. Work at INL and FRM II will continue by inserting bismuth gamma filters into the beams at NECTAR and NRAD. Further improvement is expected by new scintillation screens containing neutron converters with higher sensitivity for epithermal neutrons, and even up to higher energies. Many experiments need to be large enough to be meaningful, which is often too thick for thermal neutrons. Potential applications may include large fuel bundles, large fossils that are simply too thick for thermal neutrons to penetrate, smaller fossils in rock containing highly-attenuating minerals for thermal neutrons such as crystal water content and high iron concentration, or the study of water migration through large soil or rock specimens.

\section{References}

[1] Neutron cross sections on https://t2.lanl.gov/nis/data/endf/endfvii.1-n.html

[2] A.E. Craft, D.M. Wachs, M.A.Okuniewski, et al., Neutron radiography of irradiated nuclear fuel at Idaho National Laboratory, Physics Procedia 69 (2015) 483-490. https://doi.org/10.1016/j.phpro.2015.07.068

[3] S.H. Giegel, A.E. Craft, G.C. Papaioannou, and C.L. Pope, Neutron beam flux and energy spectrum characterization at the Neutron Radiography Reactor at Idaho National Laboratory, in this issue

[3] A. Craft, B. Schillinger, First neutron computed tomography with digital neutron imaging systems in a high-radiation environment at the $250 \mathrm{~kW}$ Neutron Radiography Reactor at Idaho, in this issue

[4] B. Schillinger, J. Krüger, A quadruple multi-camera neutron computed tomography system at MLZ, in this issue 